o.44 p. roo für die Milch- und Fettmenge sowie den Fettgehalt gering ist. Da die Interaktion auch durch Unterschiede in der genetischen Variation in den Niveaustufen verursacht sein kann, erfolgte eine Untersuchung der genetischen Parameter in jeder Stufe mit nachstehenden Ergebnissen :

\begin{tabular}{|c|c|c|c|}
\hline & \multicolumn{3}{|c|}{ Heritabilität $\left(h^{2}\right)$} \\
\hline & niedrig & mittel & hoch \\
\hline Milch-kg. & .210 & .220 & .268 \\
\hline Fett-kg. & .198 & .200 & .236 \\
\hline Fett- $\%$. & .262 & .287 & .309 \\
\hline
\end{tabular}

Es besteht die Tendenz eines leichten Anstieges der Heritabilität mit steigendem Niveau der Herden bei allen Merkmalen. Die Korrektur der Korrelation zwischen Genotypen unter verschiedenen Umwelten für die Unterschiede in den genetischen Varianzen, Niveaustufen zeigt, dass die Interaktion teilweise hierdurch verursacht ist.

\begin{tabular}{|c|c|c|}
\hline & $r_{\mathrm{G}}$ unkorrigiert & $r_{\mathrm{G}}^{\prime \prime}$ korrigiert \\
\hline Milchmenge ... & 0.965 & 0.983 \\
\hline Fettmenge $\ldots \ldots \ldots$ & 0.967 & 0.986 \\
\hline Fettgehalt $\ldots \ldots \ldots \ldots$ & 0.937 & 0.941 \\
\hline
\end{tabular}

Zum gegenwärtigen Stand des Zuchtprogrammes kann daher eine gleiche Rangierung von $\mathrm{KB}-$ Bullen im niedrigen wie hohen Leistungsniveau angenommen werden.

\title{
INTERAKTIONEN VON GENOTYP $\times$ MASTMETHODE BEI MAST- UND SCHACHTEIGENSCHAFTEN VON RINDERN
}

\section{F. Pirchner, G. Mayrhofer und J. HaAs. - Tierärztliche Hochschule, Wien (Österreich).}

Fraternale Zwillingskälber (27 Paare) wurden nach drei verschiedenen Methoden aufgezogen und gemästet : als Milchmastkälber bis zu drei Monaten, in intensiver Jungstiermast bis zu einem Jahr, als Ochsen mit zweimaliger Alpung bis zu 2 I/4 Jahren. Masse und Gewichte wurden u. a. genommen im Alter von 3 Monaten, I/2 Jahr und I Jahr. Bei der Schlachtung von allen Tieren (ausgenommen 6 Ochsen der dritten Replikation) wurden neben Ausschlachtungsprozenten die Anteile wertvoller Fleischstücke sowie die chemische Zusammensetzung des $\mathrm{m}$. long. dorsi bestimmt. Alle Werte wurden in Standardabweichungen, gerechnet vom jeweiligen Mittelwert, ausgedrückt. Es wurden die intra-class-Korrelationen für Paare sowie die genetischen Korrelationen zwischen den verschiedenen Eigenschaften in den drei Milieugruppen (Milchmast, intensive Stiermast, Ochsenmast) berechnet.

Von den Paarkorrelationen für Masse und Gewichte waren bei der I/2 Jahresgruppe (r/2 Jahr bei Ochsen und Maststieren, 3 Monate bei Milchmastkälbern) zwei von neun berechneten kleiner als 0.5 , bei der $\Upsilon$ Jahresgruppe fünf von neun. Bei den Schlachteigenschaften waren 6 von ro Paarkorrelationen kleiner als 0.5 .

Die genetischen Korrelationen waren bei drei von neun Eigenschaften der $\mathrm{x} / 2$ Jahresgruppe kleiner als 0,5 , während es bei der I Jahresgruppe fünf von neun waren. In beiden Gruppen 
waren die genetischen Korrelationen bei Gewicht hoch ( $\mathrm{I}$ oder wenig darunter), während sie bei Körpermassen im allgemeinen kleiner waren.

Die genetischen Korrelationen waren bei Schlachteigenschaften in fünf von zehn Fällen kleiner als 0,5 . Diese betrafen vor allem die chemische Zusammensetzung der Muskeln.

\section{MILCHLEISTUNG VON VIER RASSEN BEI VERSCHIEDENEM FUTTERNIVEAU}

F. Pirchner. - Tierärztliche Hochschule, Wien (Österreich).

Es wurden je ro bis 24 Kalbinnen der vier österreichischen Hauptrassen Fleckvieh, Braunvieh, Pinzgauer und Murbodner auf drei verschiedene Futtergruppen aufgeteilt. In der schwach gefütterten Gruppe wurde täglich I $\mathrm{kg}$ Kraftfutter, in der mittleren $3 \mathrm{~kg}$ und in der stark gefütterten täglich $5 \mathrm{~kg}$ Kraftfutter zusaätzlich zu Rauhfutter und Silage ohne Rücksicht auf Milchleistung gegeben. Der Fütterungsversuch begann 30 Tage postpartum und verlief über r6 Wochen. Der Futterverzehr wurde für jede Kuh bestimmt.

Die Stärkewertaufnahme hat in den drei Futtergruppen in dem Versuchszeitraum von I 6 Wochen 553, 7 10 und $805 \mathrm{~kg}$ betragen. Milchleistung, FCM Ertrag, Proteingehalt und Gewichtszunahme zeigten signifikante Futtergruppeneinflüsse und, mit Ausnahme der Gewichtszunahme, auch signifikante Rasseneinflüsse. Fettgehalt der Milch zeigte zwar Unterschiede, die ähnlich der bei Proteingehalt waren, doch erreichten sie in bezug auf Futtereinflüsse nicht statistische Signifikanz.

Durchschnittsleistungen in 16 Versuchswochen

\begin{tabular}{|c|c|c|c|c|c|c|c|}
\hline & \multicolumn{4}{|c|}{ Rassen } & \multicolumn{3}{|c|}{ Futtergruppen } \\
\hline & $F l$ & $B r$ & $P i$ & $M u$ & 1 & 2 & 3 \\
\hline Milch-kg . . . . . & 1067 & 1153 & 877 & 776 & 871 & 987 & 1047 \\
\hline FCM $\ldots \ldots \ldots \ldots$ & 1018 & 1055 & 840 & 754 & 814 & 945 & 992 \\
\hline Protein- $\% \ldots \ldots$ & 3.21 & 3.22 & 3.47 & 3.62 & 3.27 & 3.34 & 3.54 \\
\hline Zuwachs-kg ...... & 38 & 33 & 51 & 37 & 21 & 39 & 60 \\
\hline
\end{tabular}

Trotz erheblicher Unterschiede im Fütterungsniveau und deutlicher Rassenunterschiede ware keine statistisch signifikante Interaktionen zu beobachten.

\section{Divers}

\section{AI BREEDING STRATEGY FOR DUAI,-PURPOSE CATTLE POPULATIONS}

A. E. McClintock and E. P. Cunningham. - Animal Breeding and Genetics Dept., An Foras Taluntais, Dublin (Eire).

The first prerequisite in any problem of animal selection is a meaningful definition of the breeding objective. This presents special difficulties in the case of dual-purpose AI bulls, because the bull's beef and dairy genotypes will be exploited to different extents. A method 